\title{
A high-performance approach for predicting donor splice sites based on short window size and imbalanced large samples
}

Ying Zeng ${ }^{1,2}$, Hongjie Yuan ${ }^{1}$, Zheming Yuan ${ }^{1,3^{*}}$ and Yuan Chen ${ }^{4^{*}}$

\begin{abstract}
Background: Splice sites prediction has been a long-standing problem in bioinformatics. Although many computational approaches developed for splice site prediction have achieved satisfactory accuracy, further improvement in predictive accuracy is significant, for it is contributing to predict gene structure more accurately. Determining a proper window size before prediction is necessary. Overly long window size may introduce some irrelevant features, which would reduce predictive accuracy, while the use of short window size with maximum information may performs better in terms of predictive accuracy and time cost. Furthermore, the number of false splice sites following the GT-AG rule far exceeds that of true splice sites, accurate and rapid prediction of splice sites using imbalanced large samples has always been a challenge. Therefore, based on the short window size and imbalanced large samples, we developed a new computational method named chi-square decision table $\left(x^{2}-D T\right)$ for donor splice site prediction.
\end{abstract}

Results: Using a short window size of $11 \mathrm{bp}, x^{2}$-DT extracts the improved positional features and compositional features based on chi-square test, then introduces features one by one based on information gain, and constructs a balanced decision table aimed at implementing imbalanced pattern classification. With a 2000:271,132 (true sites:false sites) training set, $X^{2}$-DT achieves the highest independent test accuracy (93.34\%) when compared with three classifiers (random forest, artificial neural network, and relaxed variable kernel density estimator) and takes a short computation time (89 s). $x^{2}-D T$ also exhibits good independent test accuracy (92.40\%), when validated with BG-570 mutated sequences with frameshift errors (nucleotide insertions and deletions). Moreover, $X^{2}$-DT is compared with the long-window size-based methods and the short-window size-based methods, and is found to perform better than all of them in terms of predictive accuracy.

Conclusions: Based on short window size and imbalanced large samples, the proposed method not only achieves higher predictive accuracy than some existing methods, but also has high computational speed and good robustness against nucleotide insertions and deletions.

Reviewers: This article was reviewed by Ryan McGinty, Ph.D. and Dirk Walther.

Keywords: Donor splice site, Short window size, $x^{2}$-DT, Chi-square test, Balanced decision table

\footnotetext{
*Correspondence: zhmyuan@sina.com; 184192759@qq.com

${ }^{1}$ Hunan Engineering \& Technology Research Center for Agricultural Big Data

Analysis \& Decision-making, Hunan Agricultural University, Changsha 410128,

Hunan, China

${ }^{4}$ Hunan Provincial Key Laboratory of Crop Germplasm Innovation and

Utilization, Hunan Agricultural University, Changsha 410128, Hunan, China

Full list of author information is available at the end of the article
}

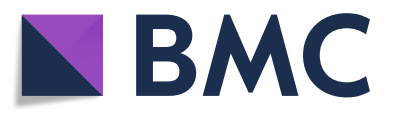

(c) The Author(s). 2019 Open Access This article is distributed under the terms of the Creative Commons Attribution 4.0 International License (http://creativecommons.org/licenses/by/4.0/), which permits unrestricted use, distribution, and

reproduction in any medium, provided you give appropriate credit to the original author(s) and the source, provide a link to the Creative Commons license, and indicate if changes were made. The Creative Commons Public Domain Dedication waiver (http://creativecommons.org/publicdomain/zero/1.0/) applies to the data made available in this article, unless otherwise stated. 


\section{Background}

The amount of genomic sequence data has increased exponentially as a result of the advancement in sequencing technology. Therefore, there is an urgent need to complete genome annotation quickly and reliably. Gene identification is an important task in genome annotation. Most eukaryotic genes consist of protein-coding regions (exons) and noncoding regions (introns), with the exons being separated by intervening introns [1]. The boundaries between exons and introns are called splice sites and are the locations where RNA splicing occurs. The $5^{\prime}$ end of an intron is a donor splice site and the $3^{\prime}$ end is an acceptor splice site. If we can accurately detect splice sites, the coding regions of DNA sequences can be located, so splice site prediction plays a key role in gene identification. Almost $99 \%$ of splice sites are canonical GT-AG pairs [2], that is, dinucleotides GT and AG for donor and acceptor splice sites, respectively. However, this strong conservation observed in splice sites is not sufficient to accurately identify them, due to the abundance of dinucleotides GT and AG appearing at non-splice site positions. We therefore face an extremely imbalanced classification task, namely, the discrimination of small numbers of true splice sites from much larger volumes of decoy positions with the dinucleotides GT and AG [3].

For splice site prediction based on machine learning approaches, the main steps are feature extraction and classifier selection or design. The extracted features are usually based on nucleotide position information [4-9], the frequency of $k$-mers $[4,6,10]$, dependence between adjacent and nonadjacent nucleotides [1, 6, 11-13], RNA secondary structure information [14-18], DNA structural properties [19], and some other attributes that can be calculated directly from sequence information [20-22]. The commonly used classifiers include support vector machine (SVM) $[1,3,5,6,10,18,23-25]$, artificial neural network (ANN) [26-29], random forest (RF) [13], and decision tree [30].

Although relatively high accuracy has been achieved with the methods currently available (e.g., the accuracy for most donor splice site prediction based on the $\mathrm{HS}^{3} \mathrm{D}$ dataset has exceeded $90 \%[6,10,12,13,19,24,31])$, further study is still necessary due to the following factors: 1) Determining a suitable window size prior to the application of any prediction method is essential [32]. Overly long window size may introduce some irrelevant features that would reduce predictive accuracy, and may take more computational time and memory space. 2) The $\mathrm{HS}^{3} \mathrm{D}$ dataset contains 2796/ 271,937 true/false donor sites (i.e., the ratio of true sites to false sites is almost $1: 100$ ). If all negative samples (false sites) are employed for building the prediction model, the huge number of training samples will increase the time complexity of some classifiers (e.g., SVM and $\mathrm{ANN})[3,33]$, and an extremely imbalanced class distribution will lead to poor predictive accuracy for some methods, for example, weighted matrix model (WMM) [9] and maximal dependency decomposition (MDD) [34]. If only a part of negative samples (e.g., 2796 negative samples [20]) are employed, predictive accuracy may be lost due to the underutilization of negative samples. 3) There are three billion DNA base pairs in the human genome, so the expected number of GT/ AG is over 187 million. This abundance means that even a subtle improvement of the total predictive accuracy would drastically increase the absolute quantity of detected real splice sites.

In this study, we developed a computational approach to predict donor splice sites based on short window size and extremely imbalanced large samples. Our method, named chi-square decision table $\left(x^{2}-\mathrm{DT}\right)$, extracts the improved positional features based on chi-square tests, combines them with the frequencies of dinucleotides, and then designs a balanced decision table to predict the test samples, which can effectively resolve the imbalanced pattern classification problem. The results show that $\chi^{2}$-DT can achieve high predictive accuracy, high computational speed, and relatively good robustness against DNA sequencing errors (nucleotide insertions and deletions).

\section{Datasets and methods}

\section{Datasets}

We collected 2796/271,928 true/false donor splice sites from the publicly available $\mathrm{HS}^{3} \mathrm{D}$ dataset [35] (http://www. sci.unisannio.it/docenti/rampone/) for the experiments, and named them $\mathrm{HS}^{3} \mathrm{D}_{\text {all }}$. Each true/false donor splice site-containing sequence has 140 nucleotides, with the conserved dinucleotide GT at the 71st and 72nd positions, and does not contain non-ACGT bases. Setting the positions of the conserved GT as 00, the upstream positions were successively labeled as $-1,-2, \ldots,-70$, whereas the downstream positions were successively labeled as $1,2, \ldots, 68$. From $\mathrm{HS}^{3} \mathrm{D}_{\text {all }}$, we randomly selected 796 true sites and 796 false sites to constitute a balanced testing set, named $\mathrm{HS}^{3} \mathrm{D}$-test ${ }_{1: 1}$, and then used the remaining sites to construct the training sets with different ratios of true sites to false sites. Additionally, to compare the performance of $x^{2}$-DT with that of other methods, we selected 2796 true sites and different numbers of false sites from $\mathrm{HS}^{3} \mathrm{D}_{\text {all }}$ to construct four datasets, namely, $\mathrm{HS}^{3} \mathrm{D}_{\mathrm{I}}, \mathrm{HS}^{3} \mathrm{D}_{\mathrm{II}}, \mathrm{HS}^{3} \mathrm{D}_{\mathrm{III}}$, and $\mathrm{HS}^{3} \mathrm{D}_{\mathrm{IV}}$.

The BG-570 dataset [36] (http://genome.crg.es/datasets/genomics96/) contains 570 human genomic DNA sequences and 570 corresponding mutated sequences. The mutated sequences were generated by introducing $1 \%$ random frameshift errors (nucleotide insertions and deletions) into the original DNA sequences. Using the BG-570 dataset, we constructed two testing sets (BG-570 orig and BG- $570_{\text {muta }}$ ) to evaluate the robustness of $X^{2}-D T$ against the frameshift errors. The extracting process of 
true/false sites in these two testing sets is described in the "Results and Discussion" section.

The numbers of true/false sites in the datasets described above are given in Table 1.

\section{Compressing $2 \times 4$ contingency table of each position with chi-square test}

Just like Pearson correlation coefficient [37] and mutual information estimators [38] that are used for identifying relationships between variables, maximal information coefficient (MIC) [39] is a novel measure proposed to capture dependences between paired variables. For a pair of data series $x$ and $y$, to calculate their MICvalue, ApproxMaxMI algorithm [39] sets $n_{x} \times n_{y}<n^{0.6}$ as the maximal grid size restriction; here, $n$ is the sample size, and $n_{x}$ and $n_{y}$ are partition bins on $x$ and $y$, respectively. Given $n=100$, the MIC score for independent paired variables should be zero, and the corresponding partition should be a $2 \times 2$ grid. However, the ApproxMaxMI algorithm tends to fall into the maximal grid size $\left(100^{0.6} \approx 16\right)$, the corresponding partition is a $2 \times 8$ grid and the corresponding MIC score is 0.24 , which leads to a nontrivial MIC score for independent paired variables under finite samples [40]. Recently, Chen et al. [40] presented the ChiMIC algorithm, which can control the excessive grid partitions of the ApproxMaxMI algorithm. Removing the maximal grid size limitation in ApproxMaxMI, ChiMIC uses a chi-square test based on a local $r \times 2$ grid to determine whether the new endpoint should be introduced. If the $p$-value of the chi-square test is lower than a given threshold, the new endpoint is introduced for partition and ChiMIC continues searching for the next optimal endpoint. If the $p$-value of the chi-square test is greater than the given threshold, the new endpoint is discarded and the process of partition is terminated. For paired independent variables with $n=100$, the MIC score

Table 1 Descriptions of various datasets

\begin{tabular}{|c|c|c|}
\hline Datasets & $\begin{array}{l}\text { Number of true } \\
\text { donor sites }\end{array}$ & $\begin{array}{l}\text { Number of false } \\
\text { donor sites }\end{array}$ \\
\hline $\mathrm{HS}^{3} \mathrm{D}_{\mathrm{all}}$ & 2796 & 271928 \\
\hline$H S^{3} D_{1}$ & 2796 & 2796 \\
\hline$H S^{3} D_{\|}$ & 2769 & 5000 \\
\hline$H S^{3} D_{I I I}$ & 2796 & 10000 \\
\hline$H S^{3} D_{I V}$ & 2796 & 15000 \\
\hline $\mathrm{HS}^{3} \mathrm{D}$-test $1: 1$ & 796 & 796 \\
\hline $\mathrm{HS}^{3} \mathrm{D}$-train $1: 1$ & 2000 & 2000 \\
\hline $\mathrm{HS}^{3} \mathrm{D}_{\text {-train }}$ 1:10 & 2000 & 20000 \\
\hline $\mathrm{HS}^{3} \mathrm{D}$-train $1: 20$ & 2000 & 40000 \\
\hline $\mathrm{HS}^{3} \mathrm{D}$-train $1: 50$ & 2000 & 100000 \\
\hline $\mathrm{HS}^{3}$ D-train 1:135 & 2000 & 271132 \\
\hline BG-570 orig & 2127 & 149039 \\
\hline BG-570 muta & 2081 & 149572 \\
\hline
\end{tabular}

calculated by ChiMIC is only about 0.06 , and the corresponding partition is a $2 \times 2$ or $2 \times 3$ grid, clearly, the grid partition produced by ChiMIC is more reasonable.

Similarly, for each position in donor splice site-containing sequences, we can build a $2 \times 4$ contingency table to respectively count the frequencies of four bases in positive and negative samples. Figure $1 \mathrm{a}$ is the $2 \times 4$ table or $2 \times 4$ grid of position 6 based on $\mathrm{HS}^{3} \mathrm{D}$-train t:135 $_{\text {. Is }}$. the $2 \times 4$ table reasonable? Could it be compressed into a $2 \times 3$ table, or even a $2 \times 2$ table? For the local $2 \times 2$ contingency table (the light gray area in Fig. 1a), the $p$-value of the chi-square test is $0.8933(>0.01)$. This indicates that the endpoint between $\mathrm{A}$ and $\mathrm{T}$ should not be introduced according to the ChiMIC algorithm. In other words, that the base at position 6 is $\mathrm{A}$ or $\mathrm{T}$ cannot provide valuable information for distinguishing positive and negative samples. Similarly, the endpoint between $\mathrm{C}$ and $\mathrm{G}$ should not be introduced. Finally, the $2 \times 4$ contingency table of position 6 is compressed into a $2 \times 2$ contingency table (see Fig. $1 \mathrm{~b}$ ).

The process of compressing the $2 \times 4$ contingency table of each position is described below. First, compress the $2 \times 4$ contingency table into six $2 \times 3$ contingency tables by merging any two different bases, and pick out the $2 \times 3$ contingency table that has the maximum chi-square value, denoted as $\max _{2 \times 3}$. Next, reconstruct a local $2 \times 2$ contingency table based on the merged bases in $\max _{2 \times 3}$ and perform a chi-square test. If the $p$-value is lower than a given threshold, $\max _{2 \times 3}$ is unreasonable and should be backtracked to the $2 \times 4$ contingency table; then, the compression process is terminated. If the $p$-value is greater than a given threshold, $\max _{2 \times 3}$ is reasonable; then, try to compress $\max _{2 \times 3}$ into a $2 \times 2$ contingency table following the two steps above. Figure 2 further illustrates the compression procedure in detail.

\section{Window size determination}

For each position in the sequences of $140 \mathrm{bp}$, we obtain a $2 \times r$ contingency Table $(2 \leq r \leq 4)$ after compression based on $\mathrm{HS}^{3} \mathrm{D}$-train ${ }_{1: 135}$; then, we perform a chi-square test with the $2 \times r$ contingency table and calculate the logarithm of the reciprocal of $p$-value, here denoted as $\log \left(p^{-1}\right)$ (see Fig. 3). Higher $\log \left(p^{-1}\right)$ values mean that the corresponding positions are more important for discriminating positives from negatives. Therefore, we determine $11 \mathrm{bp}$ (positions -3 to +8 , excluding GT at positions 00 ) as the window size for donor splice site prediction. In the following text, the study will be based on the window size of $11 \mathrm{bp}$ unless otherwise specified.

\section{Feature extraction}

From each sample (a sequence of $11 \mathrm{bp}$ in length), we extract 11 positional features and 16 compositional features. The compositional features are the frequencies of dinucleotides, which range from 0 to 10 because the 


\begin{tabular}{|c|c|c|c|c|}
\hline \multirow{2}{*}{$\begin{array}{l}\text { A } \\
\text { positive }\end{array}$} & A & $\mathrm{T}$ & $\mathrm{C}$ & G \\
\hline & 399 & 461 & 571 & 569 \\
\hline negative & 67977 & 77641 & 61049 & 64465 \\
\hline B & \multicolumn{2}{|c|}{ AT } & \multicolumn{2}{|c|}{ CG } \\
\hline positive & \multicolumn{2}{|c|}{860} & \multicolumn{2}{|c|}{1140} \\
\hline negative & & & \multicolumn{2}{|c|}{125514} \\
\hline
\end{tabular}

Fig. 1 Compressing the $2 \times 4$ contingency table of position 6. a: $2 \times 4$ contingency table of position 6 . b: $2 \times 2$ contingency table of position 6 after compression sequence sample is only $11 \mathrm{bp}$. For each feature, we can obtain a $2 \times r$ contingency Table $(2 \leq \mathrm{r} \leq 11)$ after compression; then, the status values of each feature correspond to $r$ columns of the $2 \times r$ table. For example, based on $\mathrm{HS}^{3} \mathrm{D}$-train ${ }_{1: 135}$, position 6 whose original status values are $\{A, C, G, T\}$ corresponds to a $2 \times 2$ contingency table after compression, so it has two status values as $\{\mathrm{AT}, \mathrm{CG}\}$; the frequency of dinucleotide $\mathrm{AA}$ whose original status values are $\{0,1, \ldots, 10\}$ corresponds to a $2 \times 3$ contingency table after compression, so it has three status values as $\{0,1,2-10\}$. It should be noted that, for the compositional features, only the adjacent original status values can be merged together in compression because they are sequential.

\section{Feature introduction}

Suppose the proportion of the $k^{\text {th }}$ class samples in sample set $D$ is $p_{\mathrm{k}}(k=1,2)$; then, the information entropy of $D$ is defined as:

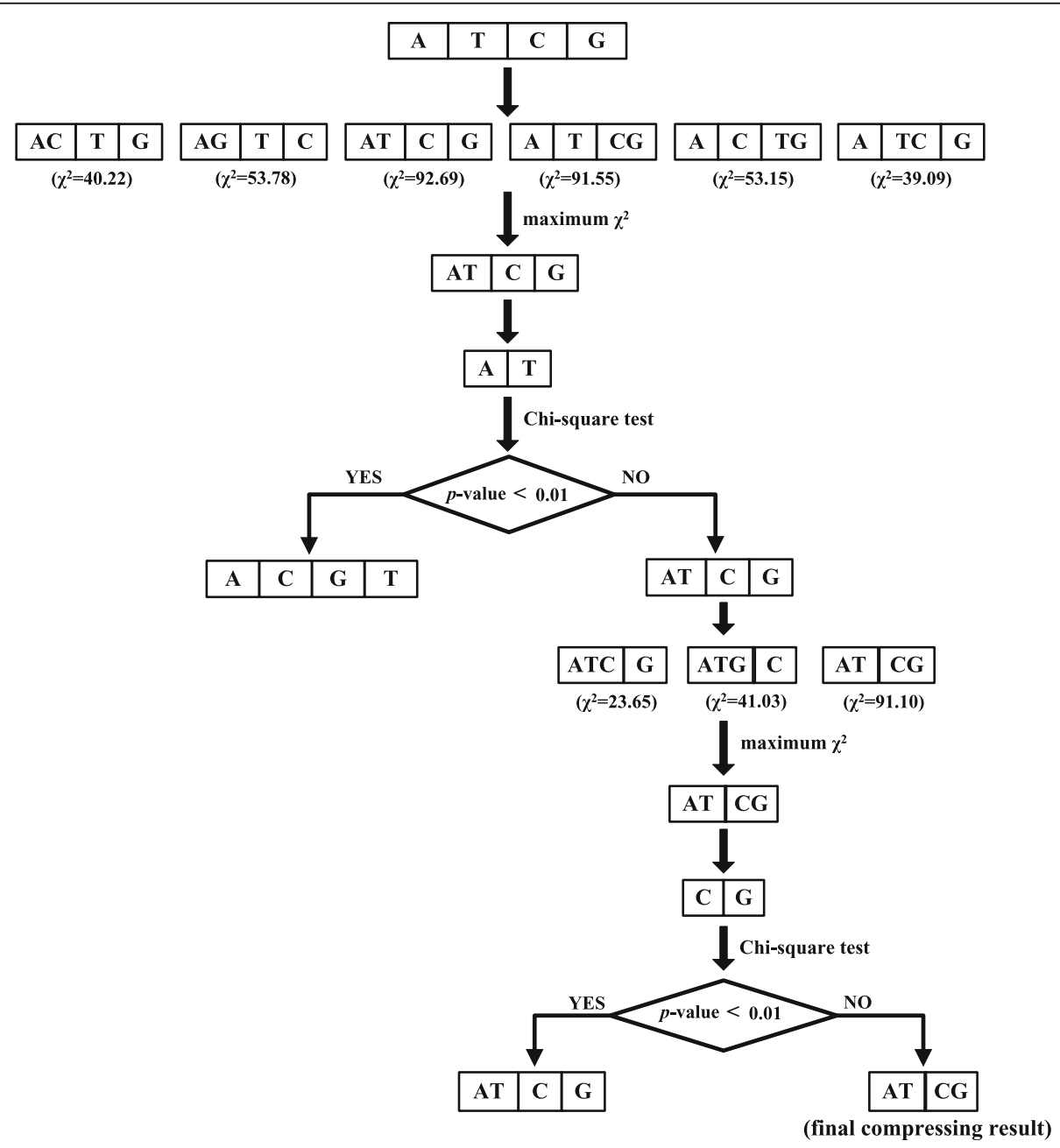

Fig. 2 Illustration of compression procedure (position 6 in $\mathrm{HS}^{3} \mathrm{D}$-train ${ }_{1: 135}$ ) 


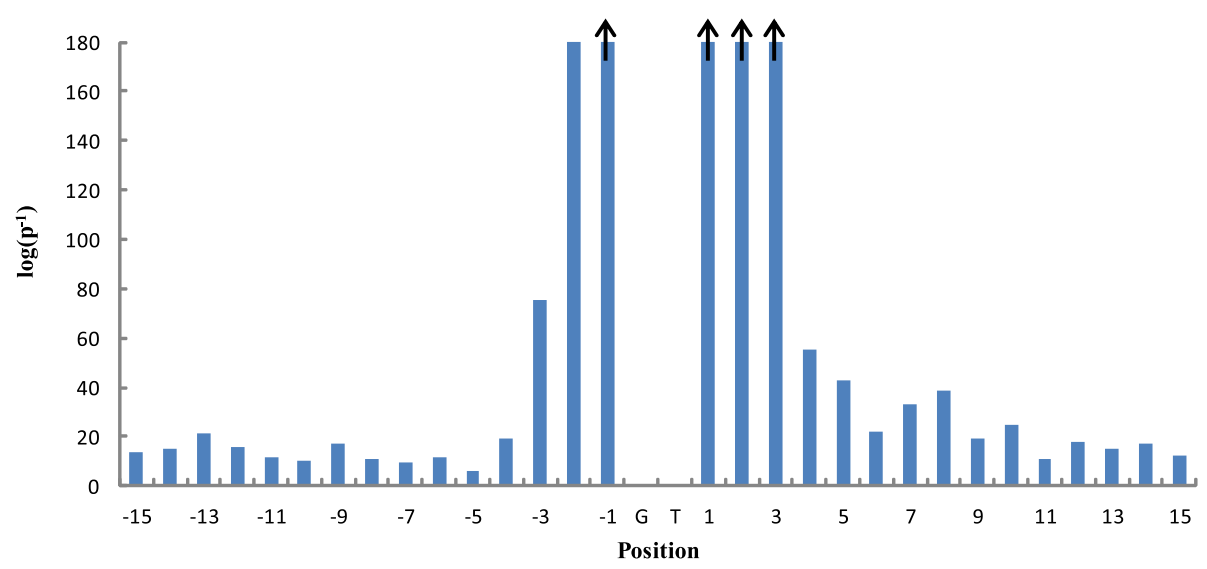

Fig. $3 \log \left(p^{-1}\right)$ values for different positions. $\uparrow$ :The columns with arrows represent that $\log \left(p^{-1}\right)$ values of the corresponding positions are higher than that of position -2 . For simplicity, we just present the $\log \left(p^{-1}\right)$ values of positions -15 to +15

$$
H(D)=-\sum_{k=1}^{2} p_{k} \log _{2} p_{k}
$$

Given a feature $X_{i}(1 \leq i \leq 27)$ that has $r(2 \leq r \leq 11)$ status values as $\left\{s_{1}, s_{2}, \ldots, s_{j}, \ldots, s_{r}\right\}$, the information gain [41] that $X_{i}$ brings for $D$ can be calculated by:

$$
\operatorname{Gain}\left(D, X_{i}\right)=H(D)-\sum_{j=1}^{r} \frac{\left|D^{j}\right|}{|D|} H\left(D^{j}\right)
$$

where $D^{j}$ represents the samples in $D$ whose $X_{i}$ takes the status value as $s_{j}(1 \leq j \leq r)$, while $H\left(D^{j}\right)$ is the information entropy of $D^{j}$.

From the features whose information gains are above the average level, we pick out the one that has the highest gain ratio to be the first introduced feature. Here, the gain ratio of $X_{i}$ is defined as:

$$
\operatorname{GainRatio}\left(D, X_{i}\right)=\frac{\operatorname{Gain}\left(D, X_{i}\right)}{I V\left(X_{i}\right)}
$$

where

$$
I V\left(X_{i}\right)=-\sum_{j=1}^{r} \frac{\left|D^{j}\right|}{|D|} \log _{2} \frac{\left|D^{j}\right|}{|D|}
$$

and $I V\left(X_{i}\right)$ is the intrinsic value of $X_{i}$.

Next, we introduce the remaining features one by one as follows.

Step 1: Under the conditions in which the introduced features have existed, further compress the $2 \times r$ contingency table of each remaining feature, in accordance with the compression process previously described. If the $r$ columns are compressed into one column, the remaining feature cannot be introduced. If the $r$ columns are not compressed into one column, the remaining feature is a candidate feature to be introduced.
Step 2: Calculate the information gain of every candidate feature. Then, from the candidate features whose information gains are above the average level, pick out the one with the highest gain ratio to be the next introduced feature.

Step 3: Repeat steps 1 and 2 until no feature can be introduced.

\section{Decision table design}

The introduced features with their status values will form various decision rules. Taking $\mathrm{HS}^{3} \mathrm{D}$-train $\operatorname{tri135}_{1}$ as an example, 27 introduced features (including 11 positional features and 16 compositional features) have formed 201 decision rules (see Additional file 1: Table S1). We separately count the numbers of positive and negative samples that conform to the decision rules, and then construct a $2 \times 201$ imbalanced decision table (Table 2). In Table 2, the decision rule " $\left(P_{3}=\mathrm{A}\right) \wedge\left(P_{-1}=\mathrm{ACT}\right) \wedge\left(0 \leq f_{G T} \leq 2\right)$ " represents position 3 taking a value of $\mathrm{A}$ and position -1 taking a value of ACT, while the frequency of dinucleotide GT takes values from 0 to 2 . Other decision rules have similar representations. Given that the number of negative samples far exceeds that of positive samples, to resolve the imbalanced pattern classification problem, we adjust the decision weight of negative samples in each column of Table 2, i.e., multiply the number of negative samples in each column by $\theta$ (here, $\theta=2000 / 271,132$ ), and then get a $2 \times 201$ balanced decision table (Table 3 ).

Table 2 Imbalanced decision table based on $\mathrm{HS}^{3}$ D-train 1:135

\begin{tabular}{lllll}
\hline Sample & Decision rule & & Total \\
\cline { 2 - 4 } & $\begin{array}{llll}\left(P_{3}=\mathrm{A}\right) \wedge\left(P_{-1}=\mathrm{ACT}\right) \wedge \\
\left(0 \leq f_{G T} \leq 2\right)\end{array}$ & $\begin{array}{l}\left(P_{3}=\mathrm{G}\right) \wedge\left(P_{-1}=\mathrm{G}\right) \wedge \\
\left(P_{1}=\mathrm{G}\right) \wedge\left(P_{2}=\mathrm{G}\right) \wedge \\
\end{array}$ & & \\
& & & \\
& & $\left(P_{4}=\mathrm{T}\right)$ & \\
& $\left(P_{-2}=\mathrm{CGT}\right)$ & \\
\hline positive & 5 & $\ldots$ & 11 & 2000 \\
negative & 47,512 & $\ldots$ & 368 & 271,132 \\
\hline
\end{tabular}


When using the balanced decision table for making decisions, suppose a test sample meets the decision rule “ $\left(P_{3}=\mathrm{A}\right) \wedge\left(P_{-1}=\mathrm{ACT}\right) \wedge\left(0 \leq f_{G T} \leq 2\right)$ ", first we assume that it is positive, replace 5 with $5+1$, and calculate the corresponding chi-square value $X_{i+}^{2}$. Then, we assume that it is negative, replace 350.5 with $350.5+1$, and calculate the corresponding chi-square value $\chi_{i-}^{2}$. If $\chi_{i+}^{2}$ $>X_{i-}^{2}$, the test sample is predicted to be positive; otherwise, it is predicted to be negative. The decision process based on an imbalanced decision table is similar.

\section{Performance evaluation}

Sensitivity (SN), specificity (SP), and the Matthew correlation coefficient (MCC) as common measures for evaluating binary classifications are defined as follows:

$$
\begin{aligned}
S N & =\frac{T P}{T P+F N} \\
S P & =\frac{T N}{T N+F P} \\
M C C & =\frac{T P \times T N-F N \times F P}{\sqrt{(T P+F N) \times(T P+F P) \times(T N+F P) \times(T N+F N)}}
\end{aligned}
$$

Here, TP, FP, TN, and FN denote the numbers of true positives, false positives, true negatives, and false negatives, respectively. SN represents the percentage of positive samples correctly predicted as true. SP represents the percentage of negative samples correctly predicted as false. MCC takes into account true and false positives and negatives, and is generally regarded as a balanced measure. However, when the class distribution of the testing set is imbalanced, the MCC value will become relatively small, which cannot really reflect the performance of a classification model.

The global accuracy index $Q^{9}[42]$ is independent of the class distribution and has been used by some researchers to evaluate the classifier performance in splice site prediction. Therefore, in this study, we choose $Q^{9}$ as the measure of global accuracy to assess predictive performance in the case of an imbalanced testing set. $Q^{9}$ is defined as follows:

$$
Q^{9}=\left(1+q^{9}\right) / 2
$$

where
The receiver operating characteristic (ROC) curve, which is widely used in evaluating the predictive accuracy of statistical predictors, is given by SN against $1-\mathrm{SP}$. When dealing with highly skewed datasets, the Precision-Recall (PR) curve can provide better insight into an algorithm's performance [43]. The areas under ROC and PR curves are denoted by AUC-ROC and AUC-PR, respectively. AUC-ROC and AUC-PR are estimated using the Davis-Goadrich method [43]. The closer the values of AUC-ROC and AUC-PR get to 1, the better the prediction model.

\section{Results and discussion}

\section{Advantage with the short window size of $11 \mathrm{bp}$}

Based on $\mathrm{HS}^{3} \mathrm{D}$-train $\mathrm{t}_{1: 1}$ and $\mathrm{HS}^{3} \mathrm{D}_{\text {-test }} \mathrm{t}_{1: 1}$, the independent tests were performed to compare the performance of $x^{2}$-DT using various window sizes. The results (Table 4) show the following: 1) Comparing with the longer window sizes (e.g., $20 \mathrm{bp}, 40 \mathrm{bp}, 138 \mathrm{bp}$ ), $\mathrm{X}^{2}$-DT with the window size of $11 \mathrm{bp}$ can achieve the higher independent test accuracy. This indicates that overly long window sizes may introduce some irrelevant sequence information, thereby reduce prediction accuracy. 2) Short window size reduces feature dimension and saves computational time. For example, using the window size of $138 \mathrm{bp}$, the feature dimension is 154 (138 positional features and 16 compositional features); however, using the window size of $11 \mathrm{bp}$, the feature dimension drops to 27 (11 positional features and 16 compositional features), and there is about a $96 \%$ decrease in the elapsed time, running in the same computer system (Intel Core i5-3320M 2.6 GHz/8 GB RAM). Therefore, we have more confident on the short window size of $11 \mathrm{bp}$. The follow-up results are all based on 11-bp-long window size.

\section{Superior performance with large extremely imbalanced dataset}

For $\mathrm{HS}^{3} \mathrm{D}$-test ${ }_{1: 1}$, we respectively used imbalanced and balanced decision tables that were built based on various $\mathrm{HS}^{3} \mathrm{D}$ training sets to make decisions. The independent test results are given in Table 5.

The results indicate the following: 1) When training sets are imbalanced, a balanced decision table can accurately predict donor splice sites. For a balanced decision table, MCC remains stable (0.847-0.867) with training sets having different positive-to-negative ratios. By contrast, for an

$$
q^{9}= \begin{cases}(T N-F P) /(T N+F P), & \text { if } T P+F N=0 \\ (T P-F N) /(T P+F N), & \text { if } T N+F P=0 \\ 1-\sqrt{2} \sqrt{[F N /(T P+F N)]^{2}+[F P /(T N+F P)]^{2},}, & \text { if } \mathrm{TP}+\mathrm{FN} \neq 0 \text { and } \mathrm{TN}+\mathrm{FP} \neq 0\end{cases}
$$


Table 3 Balanced decision table based on $\mathrm{HS}^{3}$ D-train 1:135

\begin{tabular}{lllll}
\hline Sample & Decision rule & & Total \\
\cline { 2 - 4 } & $\begin{array}{llll}\left(P_{3}=\mathrm{A}\right) \wedge\left(P_{-1}=\right. \\
\mathrm{ACT}) \wedge\left(0 \leq f_{G T} \leq 2\right)\end{array}$ & $\begin{array}{l}\left(P_{3}=\mathrm{G}\right) \wedge\left(P_{-1}=\mathrm{G}\right) \wedge\left(P_{1}=\right. \\
\wedge\left(P_{2}=\mathrm{G}\right) \wedge\left(P_{4}=\mathrm{T}\right)\end{array}$ & \\
\hline positive & 5 & $\ldots$ & 11 & 2000 \\
$\begin{array}{l}\text { negative } \\
\text { (adjusted) }\end{array}$ & 350.5 & $\ldots$ & 2.7 & 2000 \\
\hline
\end{tabular}

imbalanced decision table, MCC continually drops with an increase in negative training samples, and declines from

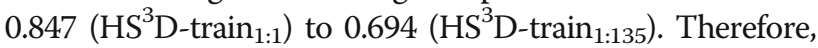
the follow-up results are produced by using balanced decision Tables. 2) Taking full advantage of training samples can improve predictive accuracy. Using a balanced decision table, MCC keeps on growing as the number of negative samples increases, and when the negative sample quantity peaks $(271,132), \mathrm{MCC}$ is at its highest (0.867).

Based on the same input features (11 positional features and 16 compositional features), $x^{2}$-DT was compared with the traditional classifiers including $\mathrm{RF}, \mathrm{ANN}$, and relaxed variable kernel density estimator (RVKDE) [44]. We selected RVKDE as a classifier for comparison because it can deliver the same level of accuracy as SVM and has lower time complexity when the training set is too large. We used Weka 3.8.1 software (https://www.cs.waikato.ac.nz/ml/ weka/index.html) and the neural network toolbox [45] of Matlab R2015a to build RF and ANN classifiers, respectively, and all of the parameters took default values. The performance comparisons still employed the inde-

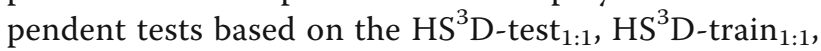
and $\mathrm{HS}^{3} \mathrm{D}$-train t:135 ; the corresponding results are given in Table 6.

The results indicate the following: 1) Using the extremely imbalanced training set, $x^{2}$-DT outperforms all of the other classifiers. As Table 6 shows, based on $\mathrm{HS}^{3} \mathrm{D}$ - $\operatorname{train}_{1: 1}$, MCC of $\mathrm{X}^{2}$-DT is 0.847 , which is comparable to those of RF, ANN, and RVKDE. In contrast, based on $\mathrm{HS}^{3} \mathrm{D}$-train $1: 135, \mathrm{MCC}$ of $\mathrm{X}^{2}$-DT rises to 0.867 , and is significantly higher than those of the other classifiers (0.248-0.353). 2) With the large training set, $x^{2}-\mathrm{DT}$ has an advantage with regard to computational speed. We ran all of the simulations on an Intel Core i5-3320M $2.6 \mathrm{GHz} / 8 \mathrm{~GB}$ RAM system. For $\mathrm{HS}^{3} \mathrm{D}$ - $\operatorname{train}_{1: 135}$, the elapsed time of $\chi^{2}$-DT was just $89 \mathrm{~s}$, while RVKDE took more than $32 \mathrm{~h}$. This speed of $\chi^{2}$-DT is due to the fact that no parameters need to be optimized.

\section{Good robustness against DNA sequencing errors}

In BG-570 dataset, setting the window size as $11 \mathrm{bp}$ (including positions -3 to -1 upstream of the conserved GT and positions +1 to +8 downstream of it, but excluding the conserved GT), we can extract 2127/149,039 true/ false donor splice site-containing sequences from 570 original DNA sequences to constitute a testing set called BG-570 orig, and extract 2081/149,572 true/false donor splice site-containing sequences from 570 mutated DNA sequences to constitute another testing set called BG-570 muta. Based on $\mathrm{HS}^{3} \mathrm{D}$-train ${ }_{1: 135}$, the independent test results respectively employing the positional features and the combination of positional and compositional features are shown in Table 7.

The MCC values in Table 7 are low (0.329-0.352) due to the highly imbalanced testing sets. To effectively assess predictive performance, the global accuracy index $Q^{9}$, which is invariant to class skew, is added for evaluation purposes. The comparative results demonstrate that: 1) The compositional features have tolerance to frameshift errors of DNA sequencing. Based on the positional features, $\mathrm{Q}^{9}$ obtained under the testing of $\mathrm{BG}-570_{\text {muta }}$ is 0.9114, lower than that under BG-570 orig $(0.9258)$. However, after adding compositional features, $\mathrm{Q}^{9}$ rises back to 0.9239 when still tested by BG- $570_{\text {muta }}$. 2) Whether or not there are frameshift errors in testing sets, $x^{2}$-DT can achieve satisfactory performance $\left(Q^{9} \geq 0.92\right)$.

\section{Better performance in comparison with existing methods}

10 -fold cross validation was applied to assess the predictive performance of $\chi^{2}$-DT, with the aim of comparing it with existing methods. To perform 10 -fold cross validation, the dataset was randomly divided into ten non-overlapping subsets of equal size. In each repetition, one subset was used as a testing set and the remaining nine subsets were used as a training set. Based on each training set, we built a balanced decision table independently. The average of ten values of predictive accuracy was used as the final accuracy. All comparisons were carried out in the $\mathrm{HS}^{3} \mathrm{D}$ datasets, and the 10-fold cross accuracy values of the methods for comparison were obtained directly from the corresponding references.

Table 4 Independent test accuracy based on various window sizes

\begin{tabular}{lllllll}
\hline Window size & Feature dimension & SN (\%) & SP (\%) & (SN + SP)/2(\%) & MCC & Time (mm:ss) \\
\hline $11 \mathrm{bp}(-3 \sim+8)$ & 27 & 93.09 & 91.58 & 92.34 & 9.847 & $00: 18$ \\
$20 \mathrm{bp}(-10 \sim+10)$ & 36 & 93.34 & 90.95 & 92.15 & 0.843 & $00: 24$ \\
$40 \mathrm{bp}(-20 \sim+20)$ & 56 & 91.33 & 91.83 & 91.58 & 0.832 & $01: 09$ \\
$138 \mathrm{bp}(-70 \sim+68)$ & 154 & 92.71 & 89.45 & 91.08 & 0.822 & $07: 18$ \\
\hline
\end{tabular}


Table $\mathbf{5}$ Independent test accuracy based on imbalanced and balanced decision tables

\begin{tabular}{|c|c|c|c|c|c|c|c|c|}
\hline \multirow[t]{2}{*}{ Training set } & \multicolumn{2}{|l|}{ SN (\%) } & \multicolumn{2}{|l|}{ SP (\%) } & \multicolumn{2}{|c|}{$(\mathrm{SN}+\mathrm{SP}) / 2(\%)$} & \multicolumn{2}{|l|}{ MCC } \\
\hline & imbal & bal & imbal & bal & imbal & bal & imbal & bal \\
\hline $\mathrm{HS}^{3} \mathrm{D}$-train $1: 1$ & 93.09 & 93.09 & 91.58 & 91.58 & 92.34 & 92.34 & 0.847 & 0.847 \\
\hline $\mathrm{HS}^{3} \mathrm{D}$-train $1: 10$ & 81.53 & 94.35 & 96.36 & 91.08 & 89.51 & 92.71 & 0.788 & 0.855 \\
\hline $\mathrm{HS}^{3} \mathrm{D}_{\text {-train }} 1: 20$ & 78.14 & 93.59 & 96.98 & 92.46 & 87.56 & 93.03 & 0.765 & 0.861 \\
\hline $\mathrm{HS}^{3} \mathrm{D}$-train $1: 50$ & 76.76 & 94.22 & 96.98 & 92.34 & 86.87 & 93.28 & 0.753 & 0.866 \\
\hline $\mathrm{HS}^{3} \mathrm{D}$-train $1: 135$ & 68.84 & 93.97 & 97.61 & 92.71 & 83.23 & 93.34 & 0.694 & 0.867 \\
\hline
\end{tabular}

imbal. Denotes imbalanced decision table and bal. denotes balanced decision table

On the one hand, $x^{2}$-DT was compared with the methods using longer window size $(\geq 100 \mathrm{bp})$, including a first-order Markov model combined with a dinucleotide-based hidden Markov model (MM1-H2MM) [31], SVM with a Bayes kernel (SVM-B) [25], and Meher's method [13]. The web server (MaLDoSS) based on Meher's method is available at http://cabgrid.res.in:8080/maldoss. The 10-fold cross accuracy of $x^{2}$-DT was calculated based on $\mathrm{HS}^{3} \mathrm{D}_{\text {all }}$. Table 8 shows that $\chi^{2}$-DT with much shorter window size can achieve better predictive performance, despite the degree of imbalance of the training set being higher.

On the other hand, $x^{2}$-DT was compared with the methods using short window size (9 bp). Maximum entropy model (MEM) [46] and SAE [12] are the typical methods for predicting donor splice sites using short window size. The web server (MaxEntScan) based on MEM is available at http://genes.mit.edu/burgelab/maxent/Xmaxentscan_scoreseq.html. The web server based on SAE is available at http://cabgrid.res.in:8080/sspred. Based on the $\mathrm{HS}^{3} \mathrm{D}$ datasets with different ratios of positive-to-negative samples (i.e., 2796:2796, 2796:5000, 2796:10000, 2796:15000), the AUC-ROC and AUC-PR values of SAE, MEM, WMM, MDD and first-order Markov model (MM1) were calculated by MaxEntScan, employing 9-bp-long window size. For comparison, we also calculated the AUC-ROC and AUC-PR values of $\chi^{2}$-DT based on $\mathrm{HS}^{3} \mathrm{D}_{\mathrm{I}}$, $\mathrm{HS}^{3} \mathrm{D}_{\mathrm{II}}, \mathrm{HS}^{3} \mathrm{D}_{\mathrm{III}}$, and $\mathrm{HS}^{3} \mathrm{D}_{\mathrm{IV}}$. The results (Table 9) showed that the predictive performance of $\chi^{2}$-DT was clearly superior to those of all of the other methods. As the degree of imbalance of the dataset increased, the AUC-PRs of all of the methods continuously declined, partly due to the fact that the evaluation indicator AUC-PR is sensitive to class skew. However, for the other methods besides $X^{2}-\mathrm{DT}$, their AUC-PRs declined more dramatically. For example, when the degree of imbalance peaked $(2796: 15,000)$, AUC-PRs of the other methods were around 0.68 , with decline of up to $28 \%$, while the AUC-PR value of $\chi^{2}$-DT was 0.85 , representing a decline of only about $10 \%$.

\section{Conclusions}

Based on the short window size of $11 \mathrm{bp}$, a high-performance method for predicting donor splice sites, called $\chi^{2}$-DT, was proposed. In terms of accuracy, $\chi^{2}$-DT is clearly superior to the methods for comparison. With regard to computational speed, $x^{2}-\mathrm{DT}$ is fast, even when using a large training set with more than 270,000 samples, because no parameters need to be optimized during model training. Furthermore, the independent test results based on the BG-570 dataset indicate that $X^{2}$-DT has relatively good robustness against frameshift errors in DNA sequencing, due to the addition of compositional features.

In future research, we plan to focus on the following: 1) We will attempt to combine more valuable features (e.g., DNA structural properties) for characterizing the candidate splice sites, in pursuit of better predictive performance. 2) When $\chi^{2}$-DT is applied to predicting acceptor splice sites, it does not further improve the predictive accuracy of existing methods, so it is necessary to devise another optimal model for acceptor sites. 3) The detection of splice sites ultimately involves identifying genes, so our overall goal is to constantly improve the proposed splice site predictor, and then use it to find genes.

\section{Reviewers' comments}

Reviewer's report 1

Ryan McGinty, Ph.D.

Table 6 Independent test accuracy based on different classifiers

\begin{tabular}{|c|c|c|c|c|c|c|c|c|}
\hline \multirow[t]{2}{*}{ Classifier } & \multicolumn{2}{|l|}{ SN (\%) } & \multirow{2}{*}{$\begin{array}{l}\text { SP (\%) } \\
\mathrm{HS}^{3} \text { D-train } \\
1: 1\end{array}$} & \multicolumn{2}{|l|}{$(\mathrm{SN}+\mathrm{SP}) / 2(\%)$} & \multicolumn{3}{|l|}{ MCC } \\
\hline & $\mathrm{HS}^{3} \mathrm{D}$-train $1: 1$ & $\mathrm{HS}^{3} \mathrm{D}$-train $1: 135$ & & $\mathrm{HS}^{3} \mathrm{D}^{- \text {train }_{1: 135}}$ & $\mathrm{HS}^{3} \mathrm{D}$-train ${ }_{1: 1}$ & $\mathrm{HS}^{3} \mathrm{D}^{- \text {train }_{1: 135}}$ & $\mathrm{HS}^{3} \mathrm{D}$-train $1: 1$ & $\mathrm{HS}^{3}$ D-train $1: 135$ \\
\hline $\mathrm{RF}$ & 94.77 & 16.58 & 91.31 & 99.87 & 93.04 & 58.23 & 0.862 & 0.297 \\
\hline ANN & 91.58 & 12.06 & 91.83 & 99.91 & 91.71 & 55.98 & 0.834 & 0.248 \\
\hline RVKDE & 96.23 & 23.37 & 88.82 & 99.50 & 92.53 & 61.43 & 0.853 & 0.353 \\
\hline$x^{2}-D T$ & 93.09 & 93.97 & 91.58 & 92.71 & 92.34 & 93.34 & 0.847 & 0.867 \\
\hline
\end{tabular}


Table $\mathbf{7}$ Independent test accuracy based on different features

\begin{tabular}{|c|c|c|c|c|c|c|}
\hline Testing set & Feature & SN (\%) & SP (\%) & $(\mathrm{SN}+\mathrm{SP}) / 2(\%)$ & MCC & $Q^{9}(\%)$ \\
\hline \multirow[t]{2}{*}{ BG-570 orig } & positional & 93.09 & 92.11 & 92.60 & 0.349 & 92.58 \\
\hline & positional+compositional & 93.51 & 92.15 & 92.83 & 0.352 & 92.70 \\
\hline \multirow[t]{2}{*}{ BG-570 muta } & positional & 90.55 & 91.77 & 91.16 & 0.329 & 91.14 \\
\hline & positional+compositional & 92.67 & 92.12 & 92.40 & 0.344 & 92.39 \\
\hline
\end{tabular}

\section{Reviewers' comments}

Reviewer summary:

Zeng, et al. have created a new computational method, $\mathrm{X} 2$-DT, for predicting gene splice donor sites that uses a very small window size ( $11 \mathrm{bp})$, is robust to a very low true/false ratio in their training data set, and runs efficiently. This method appears to perform as well or better than previous methods which were compared in this study. It would benefit the manuscript for the authors to make a clearer case for the usefulness and applicability of their tool.

Reviewer recommendations to authors:

Major suggestions: Clarify how X2-DT could be used by others and why it would be useful. The authors state that X2-DT can be used for the "prediction of splice sites in short reads generated by next-generation sequencing." However, it is never stated whether it should be applied to short reads generated from genomic sequencing or transcriptome sequencing. From the context, it would appear to be the latter, as genomic short read sequences are assembled into longer fragments and therefore the read length is irrelevant to the window size. Incidentally, there also exists a field dedicated to predicting splice site strength from genomic sequences, rather than RNA sequences. Assuming the short reads in question here are from transcriptome sequencing, the issue that the authors propose to solve can be described more clearly. In this case, the short reads should contain spliced mRNA sequences, and so the issue becomes whether there is enough sequence context on either side of the splice site to unambiguously map the splice junction without the need for computational prediction. The authors suggest that "high-throughput DNA sequencing technologies produce billions of short reads with lengths of about $50 \mathrm{bp}$ [32], while most splice site prediction methods need long sequences ( $\geq 100 \mathrm{bp}$ )."In fact, the read length of the most commonly-used platform varies from 50 to $150 \mathrm{bp}$ or more, and paired-ends can be utilized to increase the likelihood of capturing a splice junction. A
2015 study ["The impact of read length on quantification of differentially expressed genes and splice junction detection." Chhangawala, et al.] finds that "there is little difference for the detection of differential expression regardless of the read length," however, "splice junction detection significantly improves as the read length increases." From this, we can assume that someone designing a sequencing study to discover splice junctions and other features of the transcriptome would have generated $>100$ bp paired-end reads from the outset, and would not benefit greatly from the new tool presented here. However, the authors could highlight the usefulness of X2-DT in discovering splice junctions from sequencing studies where differential expression rather than transcriptome profiling was the initial aim of the study, and thus shorter reads were generated. To this end, I would suggest the authors conduct the following analyses: First, perform a parallel analysis of the same data used in Chhangawala, et al. 2015 (see above), showing the ability of X2-DT to augment splice site detection from RNA-seq data of various read lengths. For instance, does running X2-DT on $50 \mathrm{bp}$ reads find as many splice sites as $100 \mathrm{bp}$ reads without X2-DT? Does X2-DT improve on 100 bp reads at all? The authors could thus make the case for using their tool in very practical terms by showing that it is the equivalent of adding $\mathrm{N}$ bp to the sequencing read length. Next, perform a meta-analysis of the read lengths used across all RNA-seq studies, to show the magnitude of the untapped source of new splice junctions in existing RNA-seq data, which can now be found due to the unique short-window analysis of X2-DT. Combined with the above new analysis, it may be possible to estimate how many novel splice junctions can be found per transcriptome, how many transcriptomes currently exist to be analyzed, and therefore some rough estimate of the potential biological impact of this study.

Authors' response: We appreciate the detailed recommendations made by the reviewer. This study is limited to DNA sequence data generated from genomic sequencing.

Table 8 10-fold cross accuracy based on comparisons with the long-window size-based methods

\begin{tabular}{lllllll}
\hline Method & Window size (bp) & Ratio of positive-to-negative samples & SN (\%) & SP (\%) & $($ SN + SP)/2 (\%) & $Q^{9}(\%)$ \\
\hline MM1-H2MM & 140 & $2796: 27960(1: 10)$ & 93.81 & 91.69 & 92.75 & 92.63 \\
SVM-B & 140 & $2796: 27960(1: 10)$ & 94.13 & 90.99 & 92.56 & 92.39 \\
Meher's method & 102 & $2796: 53124(1: 19)$ & 88.30 & 89.40 & 88.90 & 88.80 \\
$X^{2}$-DT & 11 & $2796: 271928(1: 97)$ & 94.11 & 92.58 & 93.35 & 93.30 \\
\hline
\end{tabular}


Table 9 10-fold cross accuracy based on comparisons with the short-window size-based methods

\begin{tabular}{|c|c|c|c|c|c|c|c|c|}
\hline \multirow[t]{2}{*}{ Method } & \multicolumn{4}{|l|}{$\mathrm{AUC}-\mathrm{ROC}( \pm \mathrm{SE})$} & \multicolumn{4}{|l|}{$\mathrm{AUC}-\mathrm{PR}( \pm \mathrm{SE})$} \\
\hline & $2796: 2796$ & 2796:5000 & 2796:10000 & 2796:15000 & $2796: 2796$ & 2796:5000 & 2796:10000 & 2796:15000 \\
\hline SAE & $0.946( \pm 0.0031)$ & $0.945( \pm 0.0031)$ & $0.944( \pm 0.0030)$ & $0.945( \pm 0.0030)$ & $0.945( \pm 0.0031)$ & $0.876( \pm 0.0045)$ & $0.772( \pm 0.0055)$ & $0.682( \pm 0.0059)$ \\
\hline MEM & $0.948( \pm 0.0031)$ & $0.946( \pm 0.0031)$ & $0.947( \pm 0.0030)$ & $0.947( \pm 0.0030)$ & $0.947( \pm 0.0031)$ & $0.878( \pm 0.0045)$ & $0.773( \pm 0.0055)$ & $0.683( \pm 0.0059)$ \\
\hline MDD & $0.945( \pm 0.0031)$ & $0.942( \pm 0.0032)$ & $0.944( \pm 0.0030)$ & $0.944( \pm 0.0030)$ & $0.944( \pm 0.0031)$ & $0.872( \pm 0.0046)$ & $0.769( \pm 0.0055)$ & $0.680( \pm 0.0059)$ \\
\hline MM1 & $0.945( \pm 0.0031)$ & $0.941( \pm 0.0032)$ & $0.936( \pm 0.0032)$ & $0.941( \pm 0.0031)$ & $0.942( \pm 0.0032)$ & $0.870( \pm 0.0046)$ & $0.765( \pm 0.0056)$ & $0.679( \pm 0.0060)$ \\
\hline WMM & $0.927( \pm 0.0036)$ & $0.924( \pm 0.0036)$ & $0.924( \pm 0.0035)$ & $0.925( \pm 0.0034)$ & $0.924( \pm 0.0037)$ & $0.867( \pm 0.0046)$ & $0.703( \pm 0.0060)$ & $0.675( \pm 0.0060)$ \\
\hline$x^{2}-D T$ & $0.965( \pm 0.0023)$ & $0.969( \pm 0.0027)$ & $0.971( \pm 0.0025)$ & $0.971( \pm 0.0025)$ & $0.953( \pm 0.0030)$ & $0.932( \pm 0.0034)$ & $0.879( \pm 0.0042)$ & $0.856( \pm 0.0038)$ \\
\hline
\end{tabular}

As mentioned by the reviewer, genomic short read sequences are assembled into longer fragments before splice site prediction, so it is inappropriate to highlight the argument that $X^{2}-D T$ can predict the splice sites in short reads generated by next-generation sequencing. In the revised manuscript, we removed this inappropriate argument.

However, for our method, the use of short window size (11 bp) is necessary as far as improving prediction accuracy and simplifying the prediction model. In the revised manuscript, we discussed the benefits that 11-bp-long window size brought. Based on $\mathrm{HS}^{3} \mathrm{D}$ - train $_{1: 1}$ and $\mathrm{HS}^{3} \mathrm{D}$-test ${ }_{1: 1}$, the independent tests were performed to compare the performance of $x^{2}$-DT using various window sizes. The results (Table 10) show the following: 1) Comparing with the longer window sizes (e.g., $20 \mathrm{bp}, 40 \mathrm{bp}, 138 \mathrm{bp}$ ), $x^{2}$-DT with 11-bp-long window size can achieve higher independent test accuracy. This indicates that overly long window sizes may introduce some irrelevant sequence information, thereby reduce the prediction accuracy. 2) Short window size reduces feature dimension and saves computational time. For example, based on 138-bp-long window size, the feature dimension is 154 (138 positional features and 16 compositional features); while, as for $11 \mathrm{bp}$-long window size, the feature dimension drops to 27 (11 positional features and 16 compositional features), and there is about a $96 \%$ decrease in the elapsed time, running in the same computer system.

Additionally, as described in the results and discussion part of manuscript, $x^{2}$-DT using 11-bp-long window size was compared with several existing approaches that used longer window sizes (e.g., $140 \mathrm{bp}$ ). The results (Table 8) indicate that $\chi^{2}$-DT obtains better predictive performance.

From the results above, we have more confident on the short window size we used. Though, we still believe that correct identification depends more on the proposed method itself. As shown in the results and discussion part of manuscript, when compared with three traditional classifiers (RF, ANN, RVKDE) that are inputted the same feature vectors, our method obtains higher prediction accuracy (see Table 6); when compared with other splice site prediction approaches that also used short window size (e.g., $9 \mathrm{bp}$ ), our method is found to perform better (see Table 9). Therefore, although many computational methods have been developed presently for predicting splice sites, our method provides a supplement to the commonly used splice site prediction methods because of its high performance, and is believed to contribute to the prediction of eukaryotic gene structure.

Necessary changes have been made in the revised manuscript: Title of manuscript, Abstract, Keywords, page 4 (lines 108-110), page 5 (line 123), page 8 (lines 200-201), page 12 (lines 294-307), page 15 (lines 373,378,381-383), page 16 (line 401), titles of Tables 8 and 9; we add Table 4 and reference [32].

Minor issues:

The authors compare their work to several existing methods. While these methods are categorized and listed by their method or strategy, it would be of some practical use to know the name of each tool being compared in each table. Stylistically, I would prefer more detailed explanations of the methods that might help the study be understood by a broader audience. As written, there is a heavy prerequisite for knowledge of statistical and computational methods, including much undefined terminology. This knowledge is likely not shared by many readers interested in the biology of splicing, or with a practical need to employ the best splicing prediction program.

Table 10 Independent test accuracy based on various window sizes

\begin{tabular}{lllllll}
\hline Window size & Feature dimension & SN (\%) & SP (\%) & (SN + SP)/2(\%) & MCC & Time (mm:ss) \\
\hline $11 \mathrm{bp}(-3 \sim+8)$ & 27 & 93.09 & 91.58 & 92.34 & 9.847 & $00: 18$ \\
$20 \mathrm{bp}(-10 \sim+10)$ & 36 & 93.34 & 90.95 & 92.15 & 0.843 & $00: 24$ \\
$40 \mathrm{bp}(-20 \sim+20)$ & 56 & 91.33 & 91.83 & 91.58 & 0.832 & $01: 09$ \\
$138 \mathrm{bp}(-70 \sim+68)$ & 154 & 92.71 & 89.45 & 91.08 & 0.822 & $07: 18$ \\
\hline
\end{tabular}


Authors' response: $S V M-B, W M M, M D D, M M 1$ and $M E M$ are the conventional models for predicting splice sites, and they are often employed for comparing with new presented methods. In Table 9, the results of MEM, MDD, WMM and MM1 were obtained by executing the MaxEntScan (a web server) which is available at http://genes.mit. edu/burgelab/maxent/Xmaxentscan_scoreseq.html. Meher's method and SAE are recently developed methods for donor splice site prediction. Based on Meher's method, a web server (MaLDoSS) has been developed, and is available at http://cabgrid.res.in:8080/maldoss. The web server based on SAE can be available at http://cabgrid.res.in:8080/sspred. As per suggestion, we supplement the above contents in the revised manuscript for the convenience of practical application. As for written, we have checked and revised carefully, to avoid undefined terminology, in the hope that many readers interested in this study can understand.

Necessary changes have been made in the revised manuscript: page 15 (lines 364, 375-377, 383), page 16 (lines 384-388).

\section{Reviewer's report 2}

Dirk Walther

\section{Reviewers' comments}

Reviewer summary:

Prediction of splice-sites has been a long-standing problem in Bioinformatics and many algorithms have been developed, essentially exhausting all possible ways to formulate and solve the computational problem. Despite the many methods and their reasonable success, and despite the increased availability of transcript sequencing data which allow determining splices sites based on experimental information, this reviewer is willing to be open to new in-silico methods. Clearly, correct splice site prediction would help tremendously for genome annotation purposes.

Reviewer recommendations to authors:

(1) The authors highlight as an advantage and pose as a need to base predictions on short sequence motifs (11mers) as necessitated by the short available sequence reads from DNAseq data. Though, I would think, splices site predictions would always be applied to assembled genomes or genes, not individual reads. So for me, this is not an argument at all. The length of the k-mer should reflect what is truly necessary for correct identifications. That aside, I still believe it is interesting to see how well methods based on short k-mers can work.

Authors' response: We agree with the recommendations given by the reviewer. In the revised manuscript, we removed the inappropriate argument that $X^{2}-D T$ can predict the splice sites in short reads generated by next-generation sequencing. And we changed "short sequence" in the title to "short window size" which we thought may be more appropriate.

In this study, the use of short window size (11 bp) is necessary as far as improving prediction accuracy and simplifying the prediction model. In the revised manuscript, we discussed the benefits that 11-bp-long window size brought. Based on $\mathrm{HS}^{3} \mathrm{D}$-train ${ }_{1: 1}$ and $\mathrm{HS}^{3} \mathrm{D}$-test ${ }_{1: 1}$, the independent tests were performed to compare the performance of $\mathrm{X}^{2}$-DT using various window sizes. The results (Table 11) show the following: 1) Comparing with the longer window sizes (e.g., $20 \mathrm{bp}, 40 \mathrm{bp}, 138 \mathrm{bp}$ ), $x^{2}$-DT with 11-bp-long window size can achieve the highest independent test accuracy. This indicates that overly long window sizes may introduce some irrelevant sequence information, thereby reduce the prediction accuracy. 2) Short window size reduces feature dimension and saves computational time. For example, based on 138-bp-long window size, the feature dimension is 154 (138 positional features and 16 compositional features); while, as for 11-bp-long window size, the feature dimension drops to 27 (11 positional features and 16 compositional features), and there is about a $96 \%$ decrease in the elapsed time, when running in the same computer system.

Additionally, as described in the results and discussion part of manuscript, $x^{2}$-DT using 11-bp-long window size was compared with several existing approaches that used long window sizes (e.g., $140 \mathrm{bp}$ ). The results (Table 8) indicate that $x^{2}$-DT obtains better predictive performance.

Necessary changes have been made in the revised manuscript: Title of manuscript, Abstract, Keywords, page 4 (lines 108-110), page 5 (line 123), page 8 (lines 200-201), page 12 (lines 294-307), page 15 (lines 373,378,381-383), page 16 (line 401), titles of Tables 8 and 9; we add Table 4 and reference [32].

(2) The study reports results on donor sites only. The authors state that with regard to acceptor sites, no performance gain has been achieved, leading me to believe that performance was at least comparable. This should be discussed more - why gain for donor sites, not acceptor sites.

Table 11 Independent test accuracy based on various window sizes

\begin{tabular}{lllllll}
\hline Window size & Feature dimension & SN (\%) & SP (\%) & (SN + SP)/2(\%) & MCC & Time (mm:ss) \\
\hline $11 \mathrm{bp}(-3 \sim+8)$ & 27 & 93.09 & 91.58 & 92.34 & 0.847 & $00: 18$ \\
$20 \mathrm{bp}(-10 \sim+10)$ & 36 & 93.34 & 90.95 & 92.15 & 0.843 & $00: 24$ \\
$40 \mathrm{bp}(-20 \sim+20)$ & 56 & 91.33 & 91.83 & 91.58 & 0.832 & $01: 09$ \\
$138 \mathrm{bp}(-70 \sim+68)$ & 154 & 92.71 & 89.45 & 91.08 & 0.822 & $07: 18$ \\
\hline
\end{tabular}


Table $122 \times 9$ table for counting the number of the samples in each grid

\begin{tabular}{lllllllllll}
\hline & $0<x \leq$ & $0.05<x \leq$ & $0.29<x \leq$ & $0.55<x \leq$ & $0.57<x \leq$ & $0.62<x \leq$ & $0.69<x \leq$ & $0.71<x \leq$ & $0.84<x \leq$ & $0.85<x<1$ \\
& 0.05 & 0.29 & 0.55 & 0.57 & 0.62 & 0.69 & 0.71 & 0.84 & 0.85 \\
\hline $\begin{array}{l}0<y \leq \\
0.5\end{array}$ & 0 & 4 & 0 & 1 & 0 & 1 & 0 & 2 & 0 \\
$0.5<y<1$ & 2 & 0 & 3 & 0 & 3 & 0 & 1 & 0 & 1 & 0 \\
\hline
\end{tabular}

Also, this point should be mentioned much sooner in the manuscript than in the very last paragraph. Furthermore, the equal performance of their method relative to others should be documented.

Authors' response: We determined the 18-bp-long window size $(-17 \sim+1)$ by chi-square test, for predicting acceptor splice sites. Using 2880/28,800 true/false acceptor splice sites from $H S^{3} D$ dataset, 10-fold cross validation was applied to assess the performance of $\chi^{2}-D T$, and the predictive accuracy is: $S N=0.8901, S P=0.8751, Q^{9}=0.8826$. Based on the same dataset, $Q^{9}$ achieved by SVM-B and MM1-H2MM are 0.8951 and 0.9057, respectively, which are slightly higher than that of our method.

$x^{2}$-DT employs positional features and compositional features. While, as for acceptor sites, we found positional features and compositional features were not enough to characterize the candidate samples, maybe some other valuable features, such as DNA structural properties [19], should be involved. We are working on a new model for predicting acceptor splice sites with improved prediction accuracy, and the related researches will be reported in the forthcoming paper.

(3) The method section needs a better introduction/motivation. I had difficulties grasping the basic rationale of the method. In fact, I am not sure, I did. I could not follow the arguments with regard to "compressing that tables" at all. More explanation is needed.

Authors' response: Let's begin with maximal information coefficient (MIC) [39]. Just like Pearson correlation coefficient [37] and mutual information estimators [38] that are used for identifying relationships between variables, MIC is a novel measure proposed to capture dependences between paired variables. Giving an independent paired variables $\left\{x_{i}, y_{i}\right\}(i=1,2, \ldots 20), x_{i}, y_{i} \in(0,1)$, as shown in following:

To calculate the MIC value of $x$ and $y$, a maximum grid solution (a $2 \times 9$ grid, i.e., $y$ and $x$ are respectively partitioned as 2 bins and 9 bins) with the highest induced mutual information will be searched. And a $2 \times 9$ table (Table 12) is generated for counting the number of the samples falling into each grid.
The MIC value of $x$ and $y$ calculated based on the $2 \times 9$ grid $(2 \times 9$ table $)$ will achieve 1 , clearly, it is illogical, because MIC value should be tend to 0 for statistically independent variables. Thus, to avoid producing the nontrivial MIC values due to excessive grid partitions, ApproxMaxMI algorithm [39] sets $n^{0.6}$ as the maximal grid size restriction, here, $n$ is the sample size. Then, a $2 \times 3$ grid would be generated to partition data, and the corresponding MIC value falls to 0.31 . So the $2 \times 9$ table is compressed into a $2 \times 3$ table (Table 13).

Recently, our research group presented the ChiMIC algorithm [40] for calculating MIC value. ChiMIC uses a chi-square test based on a local $r \times 2$ grid to determine whether the new endpoint should be introduced, and removes the maximal grid size limitation in ApproxMaxMI. For the example above, the grid partition generated by ChiMIC is a $2 \times 2$ grid, and the corresponding MIC value is only about 0.11 that is more close to 0 . It means that further compressing the $2 \times 3$ grid $(2 \times 3$ table) is reasonable.

Similarly, for each position in donor splice site-containing sequences, we can build a $2 \times 4$ contingency table to respectively count the frequencies of four bases in positive and negative samples. Is the $2 \times 4$ table reasonable? Could it be compressed into a $2 \times 3$ table, or even a $2 \times 2$ table? Taking position 6 as an example, its $2 \times 4$ contingency table is finally compressed into a $2 \times 2$ contingency table, according to the ChiMIC algorithm (see Fig. 1).

Moreover, if do not compress the $2 \times 4$ contingency table of each position, we will get a $2 \times 4^{11}$ decision table after introducing 11 positional features, and with the further introduction of features, the number of columns in decision table will be grow exponentially, then the decision table would be quite sparse.

Therefore, we compressed the $2 \times \mathrm{r}$ contingency table of each feature, including positional and compositional features. And the results indicate the compression strategy is effective for correct prediction.

\begin{tabular}{lllllllllllllllllllll}
\hline$x$ & 0.08 & 0.29 & 0.71 & 0.05 & 0.69 & 0.58 & 0.55 & 0.77 & 0.06 & 0.40 & 0.84 & 0.90 & 0.57 & 0.62 & 0.01 & 0.12 & 0.46 & 0.59 & 0.98 & 0.85 \\
$y$ & 0.32 & 0.45 & 0.90 & 0.86 & 0.01 & 0.77 & 0.71 & 0.19 & 0.38 & 0.59 & 0.12 & 0.05 & 0.33 & 0.87 & 0.98 & 0.44 & 0.81 & 0.55 & 0.11 & 0.63 \\
\hline
\end{tabular}


Table $132 \times 3$ table for counting the number of the samples in each grid

\begin{tabular}{llll}
\hline & $0<x \leq 0.05$ & $0.05<x \leq 0.29$ & $0.29<x<1$ \\
\hline $0<y \leq 0.5$ & 0 & 4 & 6 \\
$0.5<y<1$ & 2 & 0 & 8 \\
\hline
\end{tabular}

Necessary changes have been made in the revised manuscript: page 6 (lines 155-157), page 7 (lines 172176); we add references [37, 38].

(4) Despite trying, I had difficulties understanding, where and how the imbalance was tested (during training or during testing or both?) Try to be more clear about it. So, in essence, I was not able to assess whether the claimed improved performance on this imbalanced problem was, in fact, achieved.

Authors' response: The number of false donor sites far exceeds that of true donor sites, e.g., the $H S^{3} D$ dataset contains 2796/271,937 true/false donor sites. If all negative samples (false sites) are employed for building the prediction model, the extremely imbalanced large training samples will lead to poor predictive results for many methods.

We give an example to explain how we resolve the imbalanced pattern classification problem. Suppose there are $87 / 1687$ positive/negative training samples, if only 2 positional features (position -1 and 3 ) are introduced and have formed 4 decision rules, we separately count the numbers of positive and negative samples that conform to the decision rules, then get a $2 \times 4$ imbalanced decision table (Table 14).

Giving a positive testing sample, suppose its position -1 and 3 both take a value of $G$, the testing sample will conform to the decision rule " $\left(P_{-1}=\mathrm{G}\right) \wedge\left(P_{3}=\mathrm{GC}\right)$ ". In Table 14, replace 11 with $11+1$, and calculate the corresponding chi-square value $\chi_{i+}^{2}$ (109.2); similarly, replace 188 with $188+1$, and calculate the corresponding chi-square value $\chi_{i-}^{2}(110.1)$. Here, $\chi_{i+}^{2}<\chi_{i-}^{2}$, so this testing sample is wrongly predicted to be negative, according to the imbalanced decision table.

Now, we adjust the decision weight of negative samples in each column, i.e., multiply the number of negative samples in each column by $87 / 1687$, and then get a balanced decision table (Table 15). In Table 15 , replace 11 with $11+$ 1 , and calculate the corresponding chi-square value $\chi_{i+}^{2}$

Table 14 Imbalanced decision table

\begin{tabular}{|c|c|c|c|c|c|}
\hline \multirow[t]{2}{*}{ Sample } & \multicolumn{4}{|l|}{ Decision rule } & \multirow[t]{2}{*}{ Total } \\
\hline & $\begin{array}{l}\left(P_{-1}=\mathrm{ACT}\right) \wedge \\
\left(P_{3}=\mathrm{AT}\right)\end{array}$ & $\begin{array}{l}\left(P_{-1}=\mathrm{ACT}\right) \wedge \\
\left(P_{3}=\mathrm{GC}\right)\end{array}$ & $\begin{array}{l}\left(P_{-1}=\mathrm{G}\right) \wedge \\
\left(P_{3}=\mathrm{AT}\right)\end{array}$ & $\begin{array}{l}\left(P_{-1}=\mathrm{G}\right) \wedge \\
\left(P_{3}=\mathrm{GC}\right)\end{array}$ & \\
\hline positive & 38 & 12 & 26 & 11 & 87 \\
\hline negative & 184 & 1026 & 289 & 188 & 1687 \\
\hline
\end{tabular}

Table 15 Balanced decision table

\begin{tabular}{llllll}
\hline Sample & \multicolumn{2}{l}{ Decision rule } & & Total \\
\cline { 2 - 5 } & $\begin{array}{l}\left(P_{-1}=\mathrm{ACT}\right) \wedge \\
\left(P_{3}=\mathrm{AT}\right)\end{array}$ & $\begin{array}{l}\left(P_{-1}=\mathrm{ACT}\right) \wedge \\
\left(P_{3}=\mathrm{GC}\right)\end{array}$ & $\begin{array}{l}\left(P_{-1}=\mathrm{G}\right) \wedge \\
\left(P_{3}=\mathrm{AT}\right)\end{array}$ & $\begin{array}{l}\left(P_{-1}=\mathrm{G}\right) \wedge \\
\left(P_{3}=\mathrm{GC}\right)\end{array}$ & \\
\hline positive & 38 & 12 & 26 & 11 & 87 \\
negative & 9.5 & 52.9 & 14.9 & 9.7 & 87 \\
\hline
\end{tabular}

(46.2); replace 9.7 with $9.7+1$, and calculate the corresponding chi-square value $\chi_{i-}^{2}$ (45.9). Here, $\chi_{i+}^{2}>\chi_{i-}^{2}$, so the testing sample is predicted to be positive. Therefore, in the case of imbalanced training set, the use of balanced decision table can correctly make decisions.

In this study, we use 2000/271,132 positive/negative samples to generate an extremely imbalanced training set

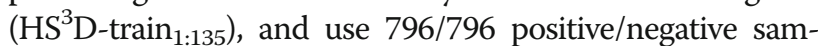
ples to generate a balanced testing set $\left(\mathrm{HS}^{3} \mathrm{D}\right.$-test $\left.{ }_{1: 1}\right)$. The independent testing results (Table 6) based on

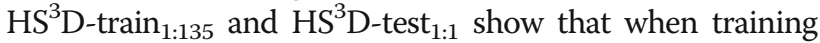
set is imbalanced, the MCC value of our method is 0.867 , while the MCC value of other traditional classifiers (RF, ANN, and RVKDE) is about $0.25-0.35$. Further, it is found in Table 5 that the MCC values obtained by balanced decision table keep on growing as the number of negative training samples increases, i.e., rise from 0.847 to 0.867 , which indicates taking full advantage of training samples could improve predictive accuracy.

Necessary changes have been made in the revised manuscript: page 10 (lines 251-253).

Minor issues

Generally, the article is well written (English-wise). Some minor mistakes need correcting. For example, use present tense in the Abstract when talking about your method and results ("The proposed method presents" (not "presented"). Check use of the definite articles.

Authors' response: Following the suggestions of reviewer, we have made language corrections, including tense and use of the definite articles.

Necessary changes have been made in the revised manuscript: Abstract, page 14 (line 346).

\section{Additional file}

Additional file 1: Table S1. The table lists 201 decision rules obtained based on $\mathrm{HS}^{3} \mathrm{D}$-train $1: 135$, and lists the number of positive and negative training samples conforming to the decision rules. (XLSX $38 \mathrm{~kb}$ )

\section{Abbreviations}

$X^{2}$-DT: Chi-square decision table; ANN: Artificial neural network; AUCPR: Areas under PR; AUC-ROC: Areas under ROC; HS3D: Homo Sapiens Splice Sites Dataset; MCC: Matthew correlation coefficient; MDD: Maximal dependency decomposition; MEM: Maximum entropy model; MIC: Maximal information coefficient; MM1: first-order Markov model; MM1-H2MM: Firstorder Markov model combined with a dinucleotide-based hidden Markov model; PR: Precision-Recall; RF: Random forest; ROC: Receiver operating characteristic; RVKDE: Relaxed variable kernel density estimator; SAE: The sum 
of absolute error; SN: Sensitivity; SP: Specificity; SVM: Support vector machine; SVM-B: SVM with a Bayes kernel; WMM: weighted matrix mode

\section{Acknowledgments}

We appreciate the thorough reading and constructive comments given by the reviewers that have significantly improved the manuscript.

\section{Funding}

This research was supported by Scientific Research Foundation of Education Office of Hunan Province, China (No.17A096, ZY); National Natural Science Foundation of China (No. 61701177, YC); Hunan Provincial Natural Science Foundation of China(2018JJ3225, YC); Science foundation open project of Hunan Provincial Key Laboratory of Crop Germplasm Innovation and Utilization(18KFXM08, YC).

\section{Availability of data and materials}

All data generated or analyzed during this study are included in this published article and its supplementary information files.

\section{Authors' contributions}

$Z Y$ conceived and designed the experiments. $Y Z$ performed the experiments. $Y Z, Y C$ and $H Y$ analyzed the data. $Y Z$ and $Z Y$ wrote the manuscript. $Y C$ contributed software coding. All authors read and approved the final manuscript.

\section{Ethics approval and consent to participate} Not applicable.

\section{Consent for publication}

All authors have read and approved the manuscript.

\section{Competing interests}

The authors declare that they have no competing interests.

\section{Publisher's Note}

Springer Nature remains neutral with regard to jurisdictional claims in published maps and institutional affiliations.

\section{Author details}

${ }^{1}$ Hunan Engineering \& Technology Research Center for Agricultural Big Data Analysis \& Decision-making, Hunan Agricultural University, Changsha 410128, Hunan, China. ${ }^{2}$ Orient Science \& Technology College, Hunan Agricultural University, Changsha 410128, Hunan, China. ${ }^{3}$ Hunan Provincial Key Laboratory for Biology and Control of Plant Diseases and Insect Pests, Hunan Agricultural University, Changsha 410128, Hunan, China. ${ }^{4}$ Hunan Provincial Key Laboratory of Crop Germplasm Innovation and Utilization, Hunan Agricultural University, Changsha 410128, Hunan, China.

Received: 15 December 2018 Accepted: 18 March 2019 Published online: 11 April 2019

\section{References}

1. Baten AKMA, Chang BCH, Halgamuge SK, Li J. Splice site identification using probabilistic parameters and SVM classification. BMC Bioinformatics. 2006;7:15.

2. Burset M, Seledtsov IA, Solovyev W. Analysis of canonical and noncanonical splice sites in mammalian genomes. Nucleic Acids Res. 2000; 28(21):4364-75

3. Sören S, Schweikert G, Philips P, Behr J, Rätsch G. Accurate splice site prediction using support vector machines. BMC Bioinformatics. 2007; 8(Suppl 10):7.

4. Degroeve $S$, Saeys $Y$, Baets BD, Rouzé $P$, Peer YVD. SpliceMachine: predicting splice sites from high-dimensional local context representations. Bioinformatics. 2005;21(8):1332-8.

5. Huang J, Li T, Chen K, Wu J. An approach of encoding for prediction of splice sites using SVM. Biochimie. 2006;88(7):929.

6. Li JL, Wang LF, Wang HY, Bai LY, Yuan ZM. High-accuracy splice site prediction based on sequence component and position features. Genet Mol Res. 2012;11(3):3432-51.

7. Nasibov E, Tunaboylu S. Classification of splice-junction sequences via weighted position specific scoring approach. Comput Biol Chem. 2010; 34(5-6):293-9.
8. Pertea M, Lin XY, Salzberg SL. GeneSplicer: a new computational method for splice site prediction. Nucleic Acids Res. 2001;29(5):1185-90.

9. Staden R. Computer methods to locate signals in nucleic acid sequences. Nucleic Acids Res. 1984;12(2):505.

10. Wei D, Zhang HL, Wei YJ, Jiang QS. A novel splice site prediction method using Support Vector Machine. J Comput Inf Syst. 2013;20:8053-60.

11. Arita M, Tsuda $K$, Asai K. Modeling splicing sites with pairwise correlations. Bioinformatics. 2002;18(Suppl 1):27-34.

12. Meher PK, Sahu TK, Rao AR, Wahi SD. A statistical approach for 5'splice site prediction using short sequence motifs and without encoding sequence data. BMC Bioinformatics. 2014;15(1):362.

13. Meher PK, Sahu TK, Rao AR. Prediction of donor splice sites using random forest with a new sequence encoding approach. Biodata Min. 2016;9(1):4.

14. Marashi SA, Goodarzi H, Sadeghi M, Eslahchi C, Pezeshk H. Importance of RNA secondary structure information for yeast donor and acceptor splice site predictions by neural networks. Comput Biol Chem. 2006; 30(1):50-7.

15. Patterson DJ, Yasuhara K, Ruzzo WL. Pre-mRNA secondary structure prediction aids splice site prediction. Pac Symp Biocomput. 2002;7:223-34.

16. Buratti $E$, Baralle FE. Influence of RNA secondary structure on the pre-mRNA splicing process. Mol Cell Biol. 2004:24:10505-14.

17. Mareshi S, Eslahchi C, Pezechk H. Impact of RNA structure on the prediction of donor and acceptor splice sites. BMC Bioinformatics. 2008;7:297.

18. Sun YF, Fan XD, Li YD. Identifying splicing sites in eukaryotic RNA: support vector machine approach. Comput Biol Med. 2003;33(1):17-29.

19. Zuo YC, Zhang PF, Li L. Sequence-specific flexibility organization of splicing flanking sequence and prediction of splice sites in the human genome. Chromosom Res. 2014:22(3):321-34.

20. Chen W, Feng PM, Lin H, Chou KC. iSS-PseDNC: identifying splicing sites using Pseudo dinucleotide composition. Biomed Res Int. 2014;2014:623149.

21. Hebsgaard SM, Korning P, Brunak S. Splice site prediction in Arabidopsis thaliana pre-mRNA by combining local and global sequence information. Nucleic Acids Res. 1996;24(17):3439-52.

22. Zhang XH, Heller KA, Hefter I, Leslie CS, Chasin AL. Sequence information for the splicing of human pre-mRNA identified by support vector machine classification. Genome Res. 2003;13(12):2637.

23. Baten A, Halgamuge $S$, Chang B. Fast splice site detection using information content and feature reduction. BMC Bioinformatics. 2008;9(Suppl 12):8.

24. Maji S, Garg D. Hybrid approach using SVM and MM2 in splice site junction identification. Curr Bioinforma. 2014;9:76-85.

25. Zhang Y, Chu CH, Chen YX, Zha HY, Ji X. Splice site prediction using support vector machines with a Bayes kernel. Expert Syst Appl. 2006; 30(1):73-81.

26. Ho LS, Rajapakse JC. Splice site detection with a higher-order Markov mode implemented on a neural network. Genome Inform. 2003;14:64-72.

27. Rajapakse JC, Ho LS. Markov encoding for detecting signals in genomic sequences. IEEE/ACM Trans Comput Biol Bioinformatics. 2005;2(2):131.

28. Liu L, Ho YK, Yau S. Prediction of primate splice site using inhomogeneous Markov chain and neural network. DNA Cell Biol. 2007;26(7):477-83.

29. Tripti N, Shailendra S, Neelam G. Splice site detection in DNA sequences using probabilistic neural network. Int J Comput Appl. 2013;76(4):1-4.

30. Huang YF, Liang CP, Liou SW. Intron identification approaches based on weighted features and fuzzy decision trees. Comput Biol Med. 2011; 42:112-22.

31. Zhang Q, Peng Q, Li K, Kang X, Li J. Splice sites detection by combining Markov and hidden Markov model. In: The 2nd international conference on biomedical engineering and informatics; Tianjin, China; 2009. p. 1-5.

32. Meher PK, Sahu TK, Rao AR, Wahi SD. Determination of window size and identification of suitable method for prediction of donor splice sites in rice (Oryza sativa) genome. J Plant Biochem Biotechnol. 2015;24(4):385-92.

33. Zhang Q, Peng Q, Zhang Q. Splice sites prediction of human genome using length-variable Markov model and feature selection. Expert Syst Appl. 2010; 37:2771-82

34. Burge C, Karlin S. Prediction of complete gene structure in human genomic DNA. J Mol Biol. 1997;268(1):78-94.

35. Pollastro P, Rampone S. HS3D, a dataset of homo sapiens splice regions, and its extraction procedure from a major public database. International Journal of Modern Physics C. 2002;13(8):1105-17.

36. Burset M, Guigó R. Evaluation of gene structure prediction programs. Genomics. 1996;34(3):367. 
37. Pearson K. Notes on the history of correlation. Biometrika. 1920;13(1):25-45.

38. Moon YI, Rajagopalan B, Lall U. Estimation of mutual information using kernel density estimators. Phys Rev E Stat Phys Plasmas Fluids Relat Interdiscip Topics. 1995;52(3):2318-21.

39. Reshef DN, Reshef YA, Finucane HK, Grossman SR, McVean G, Turnbaugh PJ. Detecting novel associations in large data sets. Science. 2011;334:1518-24.

40. Chen Y, Zeng Y, Luo F, Yuan Z. A new algorithm to optimize maximal information coefficient. PLoS One. 2016;11(6):e0157567.

41. Shang C, Li M, Feng S, Jiang Q, Fan J. Feature selection via maximizing global information gain for text classification. Knowl-Based Syst. 2013;54:298-309.

42. Zhang $C T$, Zhang R. Evaluation of gene-finding algorithms by a contentbalancing accuracy index. J Biomol Struct Dyn. 2002;19(6):1045-52.

43. Davis J, Goadrich M. The relationship between Precision-Recall and ROC curves. In: Proceedings of the 23rd international conference on machine learning. Pittsburgh, Pennsylvania, United States, ACM. pp 233-240. http:// dx.doi.org/10.1145/1143844.1143874.

44. Oyang YJ, Hwang SC, Ou YY, Chen CY, Chen ZW. Data classification with radial basis function networks based on a novel kernel density estimation algorithm. IEEE Trans Neural Netw. 2005;16(1):225-36

45. Raida Z. Modeling EM structures in the neural network toolbox of MATLAB. IEEE Antennas Propagation Mag. 2002;44(6):46-67.

46. Yeo G, Burge CB. Maximum entropy modeling of short sequence motifs with applications to RNA splicing signals. J Comput Biol. 2004;11:377-94.

Ready to submit your research? Choose BMC and benefit from:

- fast, convenient online submission

- thorough peer review by experienced researchers in your field

- rapid publication on acceptance

- support for research data, including large and complex data types

- gold Open Access which fosters wider collaboration and increased citations

- maximum visibility for your research: over $100 \mathrm{M}$ website views per year

At $\mathrm{BMC}$, research is always in progress.

Learn more biomedcentral.com/submissions 